\title{
Recapitulation of erythropoiesis in congenital dyserythropoietic anemia type I (CDA-I) identifies defects in differentiation and nucleolar abnormalities
}

Haematologica 2021

Volume 106(11):2960-2970

\section{Correspondence:}

CAROLINE SCOTT

caroline.scott@imm.ox.ac.uk

VERONICA BUCKLE

veronica.buckle@imm.ox.ac.uk

Received: May 20, 2020.

Accepted: September 17, 2020.

Pre-published: October 29, 2020.

https://doi.org/10.3324/haematol.2020.260158

(C)2021 Ferrata Storti Foundation

Material published in Haematologica is covered by copyright. All rights are reserved to the Ferrata Storti Foundation. Use of published material is allowed under the following terms and conditions:

https://creativecommons.org/licenses/by-nc/4.0/legalcode. Copies of published material are allowed for personal or internal use. Sharing published material for non-commercial purposes is subject to the following conditions:

https://creativecommons.org/licenses/by-nc/4.0/legalcode, sect. 3. Reproducing and sharing published material for commercial purposes is not allowed without permission in writing from the publisher.

\section{Caroline Scott, ${ }^{1}$ Damien J. Downes, ${ }^{1}$ Jill M. Brown, ${ }^{1}$ Robert A. Beagrie, ${ }^{1}$ Aude-Anais Olijnik, ${ }^{1}$ Matthew Gosden, ${ }^{1}$ Ron Schwessinger, ${ }^{1}$ Christopher A. Fisher, ${ }^{1}$ Anna Rose, ${ }^{1}$ David J.P Ferguson, ${ }^{2}$ Errin Johnson, ${ }^{3}$ Quentin A. Hill, ${ }^{4}$ Steven Okoli, ${ }^{5}$ Raffaele Renella, ${ }^{6}$ Kate Ryan, ${ }^{7}$ Marjorie Brand, ${ }^{8}$ Jim Hughes, ${ }^{1}$ Noemi B.A. Roy, ${ }^{9,10}$ Douglas R. Higgs, ${ }^{10}$ Christian Babbs ${ }^{1}$ and Veronica J. Buckle ${ }^{1}$}

${ }^{1}$ MRC Weatherall Institute of Molecular Medicine, Oxford University, Oxford, UK; ${ }^{2}$ Ultrastructural Morphology Group, NDCLS, John Radcliffe Hospital, Oxford, UK; ${ }^{3}$ Sir William Dunn School of Pathology, Oxford University, Oxford, UK; ${ }^{4}$ Leeds Teaching Hospital NHS Trust, Leeds, UK; ${ }^{5}$ Imperial College, The Commonwealth Building, The Hammersmith Hospital, Du Cane Rd, London, UK; ${ }^{6}$ Pediatric Hematology-Oncology Research Laboratory, CHUV-UNIL Lausanne, Lausanne, Switzerland; ${ }^{7}$ Department of Hematology, Manchester Royal Infirmary, Manchester, UK; ${ }^{8}$ Sprott Center for Stem Cell Research, Ottawa Hospital Research Institute, Ottawa, Ontario, Canada; ${ }^{9}$ Department of Hematology, Oxford University Hospitals NHS Trust, Churchill Hospital, Headington, and NIHR Biomedical Research Centre, Oxford, UK and ${ }^{10}$ National Institute of Health Research Oxford Biomedical Research Center, Oxford, UK

\section{ABSTRACT}

The investigation of inherited disorders of erythropoiesis has elucidated many of the principles underlying the production of normal red blood cells and how this is perturbed in human disease. Congenital dyserythropoietic anemia type 1 (CDA-I) is a rare form of anemia caused by mutations in two genes of unknown function: CDAN1 and CDIN1 (previously called C15orf41), whilst in some cases, the underlying genetic abnormality is completely unknown. Consequently, the pathways affected in CDA-I remain to be discovered. In order to enable detailed analysis of this rare disorder we have validated a culture system which recapitulates all of the cardinal hematological features of CDA-I, including the formation of the pathognomonic 'spongy' heterochromatin seen by electron microscopy. Using a variety of cell and molecular biological approaches we discovered that erythroid cells in this condition show a delay during terminal erythroid differentiation, associated with increased proliferation and widespread changes in chromatin accessibility. We also show that the proteins encoded by CDAN1 and CDIN 1 are enriched in nucleoli which are structurally and functionally abnormal in CDA-I. Together these findings provide important pointers to the pathways affected in CDA-I which for the first time can now be pursued in the tractable culture system utilized here.

\section{Introduction}

Many key discoveries in the process of erythropoiesis have come from identification and analysis of individuals with forms of inherited anemia. ${ }^{1-4}$ In some cases, because of the rarity of the disease and limited access to primary erythroid progenitors and precursors, progress can only be made by developing appropriate models of the disease or, ideally, methods that only require access to peripheral blood. Here we have utilised such an approach to study congenital dyserythropoietic anemia type 1 (CDA-I).

CDA-I is a rare autosomal recessive disease associated with ineffective erythropoiesis and macrocytic anemia. Disease severity is commonly mild (not necessitating treatment) to moderate (requiring Interferon or occasional blood transfusion) but patients with severe disease can be transfusion-dependent from birth or even 
present as hydrops fetalis. ${ }^{5,6}$ Light microscopy reveals abnormalities in erythroblast nuclei, including binucleate cells and inter-nuclear bridging. ${ }^{5}$ A diagnostic feature of CDA-I is the "Swiss-cheese" or spongy pattern of abnormal chromatin in up to $50 \%$ of erythroblasts obtained from bone marrow aspirates, visualised using transmission electron microscopy (TEM). ${ }^{6}$ In $~ 90 \%$ of patients, biallelic mutations in CDAN1 (encoding Codanin-1) or CDIN1 (previously C15orf41) (encoding CDIN1) are causative, 7,8 with the genetic basis of the remaining $10 \%$ of patients yet to be determined. Both Codanin-1 and CDIN1 are widely expressed and appear to be essential to life $e^{5}$ but their precise functions are unclear. CDIN1 comprises a helix-turn-helix binding domain and a predicted nuclease domain ${ }^{8}$ whilst Codanin-1 has sequence similarity with a scaffold protein, CNOT1, involved in mRNA stability and translational control. ${ }^{9}$ The two proteins form a complex where Codanin-1 is required for stability of CDIN1 ${ }^{9-11}$ and both directly interact with the histone chaperone ASF1. ${ }^{12-14}$ CDA-I is predominantly an erythroid-restricted disease but most of the structural and functional assessments of Codanin-1 and CDIN1 have been performed in non-erythroid cells and some characteristics described for the proteins are not recapitulated in patient-derived erythroblasts. ${ }^{9,11}$ Here we analyze the distribution and role of these proteins in erythroid cells.

Differentiation of $\mathrm{CD}_{3} 4^{+}$hematopoietic stem and progenitor cells (HSPC) from peripheral blood has been used to study normal erythropoiesis ${ }^{15-19}$ and to elucidate disease mechanisms in a number of hematological disorders including Diamond Blackfan anemia (DBA), ${ }^{2,3}$ hereditary spherocytosis, ${ }^{20}$ congenital dyserythropoietic anemia type $\mathrm{II}^{21}$ and myelodysplastic syndrome (MDS). ${ }^{16}$ In order to fully understand the defects that arise in patients who do not generate sufficient mature cells, any culture system must recapitulate terminal erythropoiesis through to enucleation and erythroblasts from controls and patients should be stage matched. ${ }^{22}$ Here we use an ex vivo threephase culture system ${ }^{15}$ (broadly expansion, differentiation and enucleation) whereby $\mathrm{CD} 34^{+} \mathrm{HSPC}$, obtained from peripheral blood of healthy donors and patients with TEM-confirmed CDA-I, are successfully differentiated into reticulocytes. We use immunophenotyping by fluorescence activated cell sorting (FACS), morphological assessment, single cell proteomics using mass cytometry time of flight (CyTOF) and assay for transposase-accessible chromatin using sequencing (ATAC-seq) to enable direct comparisons of cell populations.

This approach successfully recapitulates the pathognomonic feature of 'spongy' heterochromatin in patient erythroblasts. We find a delay in terminal erythroid differentiation and increased proliferation of CDA-I erythroblasts, associated with widespread changes in chromatin accessibility. We demonstrate that CDIN1 and Codanin-1 are enriched in nucleoli, which are structurally and functionally abnormal in CDA-I. These findings provide important indicators to the pathways affected in CDA-I, which can now be pursued in the tractable model of erythropoiesis utilised here.

\section{Methods}

\section{Patient recruitment}

Subjects were referred for next-generation sequencing through the Oxford Molecular Diagnostic Center. If a molecular diagnosis of CDA-I was made, patient consent was obtained for entry into this research study approved by the Wales Research Ethics Committee (REC5) (13/WA/0371), with written consent and compliance with the Declaration of Helsinki.

\section{Isolation and differentiation of CD34 ${ }^{+}$hematopoietic stem and progenitor cells}

Peripheral blood mononuclear cells were isolated from $50 \mathrm{~mL}$ of EDTA anti-coagulated peripheral blood from three healthy donors and ten CDA-I patients using Histopaque. The CD34 HSPC were extracted with the Human CD34 Microbead Kit (Miltenyi Biotec), according to the manufacturer's instructions. $1 \times 10^{5}$ frozen CD $34^{+}$HSPC were recovered into Phase I media of a three-phase protocol ${ }^{15}$ (see Online Supplementary Figure S2A and the Online Supplementary Appendix) and monitored by cytospin (see the Online Supplementary Appendix) and FACS (Online Supplementary Table S1; Online Supplemental Figure S2).

\section{Transmission electron microscopy}

$5 \times 10^{6}$ staged intermediate erythroblasts were prepared for TEM as previously described. ${ }^{4,23}$

\section{Iso-electric focusing}

$1 \times 10^{6}$ cultured erythroblasts were analysed by iso-electric focusing (IEF) (see the Online Supplementary Appendix).

\section{Real-time quantitative polymerase chain reaction for globins}

RNA was extracted using a Tri-reagent protocol and real-time quantitative polymerase chain reaction (RT-qPCR) conducted with commercial TaqMan assays (Online Supplementary Table S2).

\section{Chromatin accessibility and NF-E2 chromatin immunoprecipitation sequencing}

ATAC-seq was performed as previously described. ${ }^{24,25}$ NF-E2 chromatin immunoprecipitation sequencing (ChIP-seq) was conducted on $5 \times 10^{6}$ day 10 erythroblasts with previously described modifications ${ }^{26}$ using rabbit anti-NFE2 (4 $\mu \mathrm{g}$, sc-22827X; Santa Cruz discontinued). For ATAC-seq library preparation and analysis see the Online Supplementary Appendix.

\section{Antibody labeling, barcoding and mass cytometry for mass cytometry time of flight}

Samples were prepared and analysed for CyTOF as previously described $^{27}$ (Online Supplementary Table S6). Subsampled events were concatenated for uniform manifold approximation and projection (UMAP) ${ }^{28}$ See the Online Supplementary Appendix.

\section{Data availability}

Sequencing data generated for this work is available on the Gene Expression Omnibus (GSE125175).

\section{Immunofluorescence}

Cells were washed and processed essentially as described previously. ${ }^{29}$ See the Online Supplementary Appendix.

\section{Fluorescence in situ hybridization}

Fluorescence in situ hybridization (FISH) probes used were p7.1 (covering most of the rDNA array) and BAC CT476834 (demarcating perinucleolar heterochromatin) and were kindly gifted by Prof B. McStay. ${ }^{30}$ Probes were labeled with Cy3-dUTP (GE Healthcare) or indirectly with digoxygenin-11-dUTP (Roche). ${ }^{31}$ See the Online Supplementary Appendix. 
A

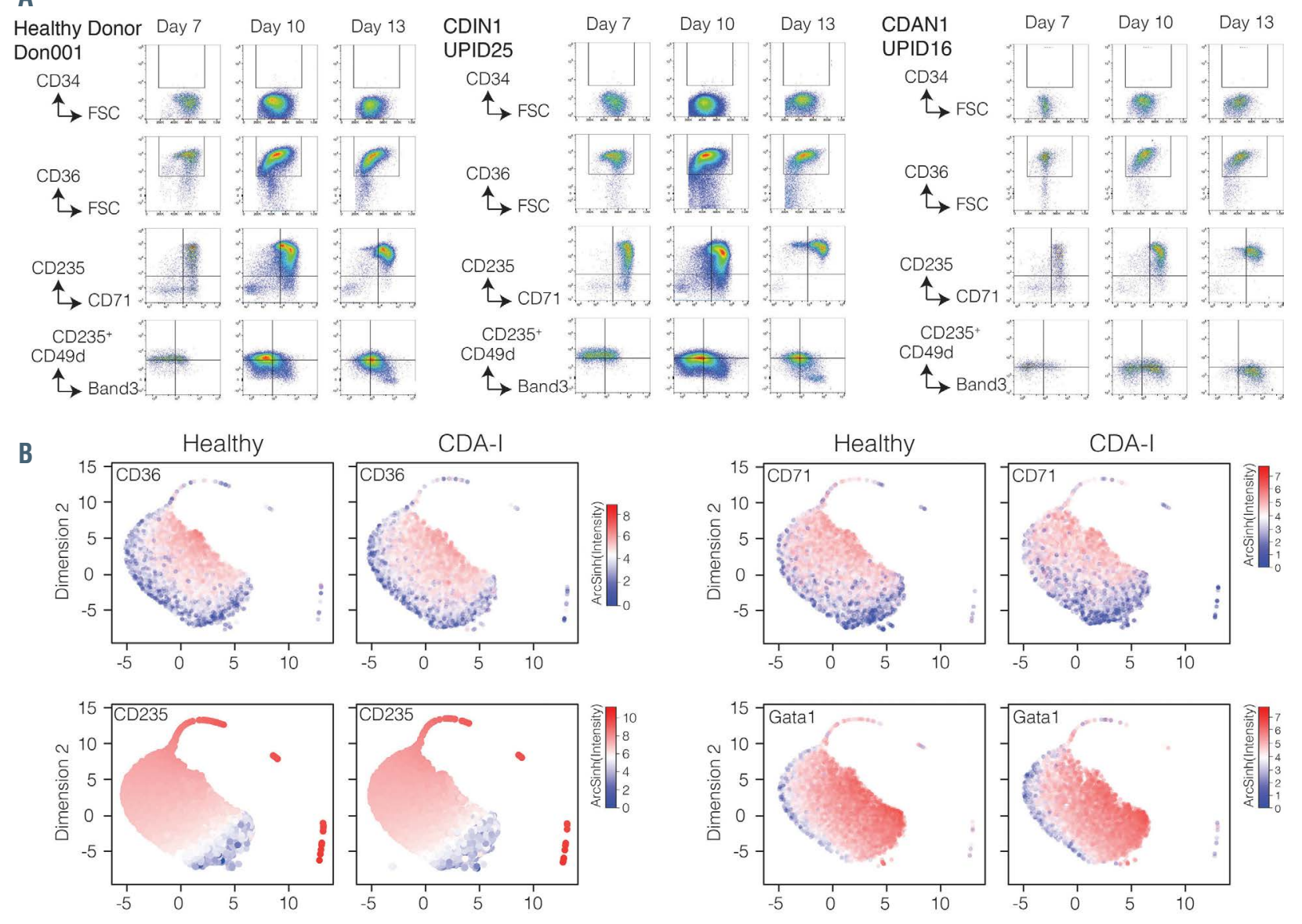

Figure 1. Differentiating erythroblasts from healthy donors and CDA-I patients are broadly equivalent by immunophenotyping. (A) Representative fluorescence activated cell sorting profiles of cultured erythroblasts from a healthy donor, CDA-I patient with a mutation in CDIN1 and CDA-I patient with a mutation in CDAN1. (B) Uniform manifold approximation and projection (UMAP) plots showing mass cytometry time of flight (CyTOF) data from healthy donors ( $n=3$ ) and CDIN1-patient derived erythroblasts $(n=3)$ at day 11 of differentiation for the erythroid markers CD235 (glycophorin A), CD36 (Scavenger receptor), CD71 (Transferrin receptor) and transcription factor Gata1. There is no difference in the clustering patterns observed between healthy donors and CDIN1 patients for any of the 25 markers tested (Online Supplementary Table S6).

\section{Ethynyl uridine labeling and analysis}

RNA transcripts were labeled by 5ethynyl uridine $(\mathrm{EU})$ incorporation (1 $\mathrm{mM}$ for 30 minutes or 2 hours) and detected by click chemistry with Alexa488 ${ }^{\circledR}$ azide using Click-i ${ }^{\circledR}$ RNA imaging Kit (ThermoFisher Scientific). Quantitation of EU was performed using FIJI. ${ }^{32}$ All images were acquired using standardized settings and maximum-intensity projected. Mean EU intensities were quantitated using a nuclear mask demarcated by DAPI.

\section{Results}

\section{CDA-I patients}

To date, approximately 60 mutations have been reported in CDAN1 and CDIN1, $1,7,8,33$ and six mutational hotspots have been identified in the CDAN1 gene. ${ }^{10}$ There are often differences in the severity of the disease between individuals, even for those with identical mutations. $^{34}$ We examined erythropoiesis in ten CDA-I patients (Online Supplementary Figure S1A; Online Supplementary Table S8). These patients (excluding those receiving regular blood transfusion or venesections) have hemoglobin $(\mathrm{Hb})$ levels and mean cell volumes (MCV) within the normal range (Online Supplementary Figure 1B), consistent with $\sim 30 \%$ of clinical cases ${ }^{6}$ but tend to have higher mean cell hemoglobin $(\mathrm{MCH})$, an increased red cell distribution width (RDW) and a reduced red cell count (RBC) compared to healthy donors (Online Supplementary Figure $S 1 B$ ). In one patient (UPID6) with CDA-I, confirmed by TEM, a potentially pathogenic homozygous variant was identified in CDAN1 although the allele frequency for this mutation is $>1 \%$ in specific populations. Data from this patient was included in the CDAN1 mutation group.

\section{Establishing a suitable model system using peripheral blood-derived CD34 ${ }^{+}$hematopoietic stem and progenitor cells}

We initially validated a three-phase ex vivo culture system $^{15}$ for differentiation of CD $34^{+}$HSPC obtained from the peripheral blood of healthy donors (Online Supplementary Figures S2 and S3). In addition to the morphological assessment of cultured erythroblasts (Online Supplementary Figure S2B), we characterized their chromatin landscape, globin gene expression profile and the expression of erythroid proteins and transcription factors, to comprehensively evaluate differentiation status (Online Supplementary Figure S4). Immunophenotyping revealed the expected gain of glycophorin A (CD235a) and transferrin receptor (CD71), which typically occurred by day 7 (Online Supplementary Figure S2C). As maturation pro- 
A

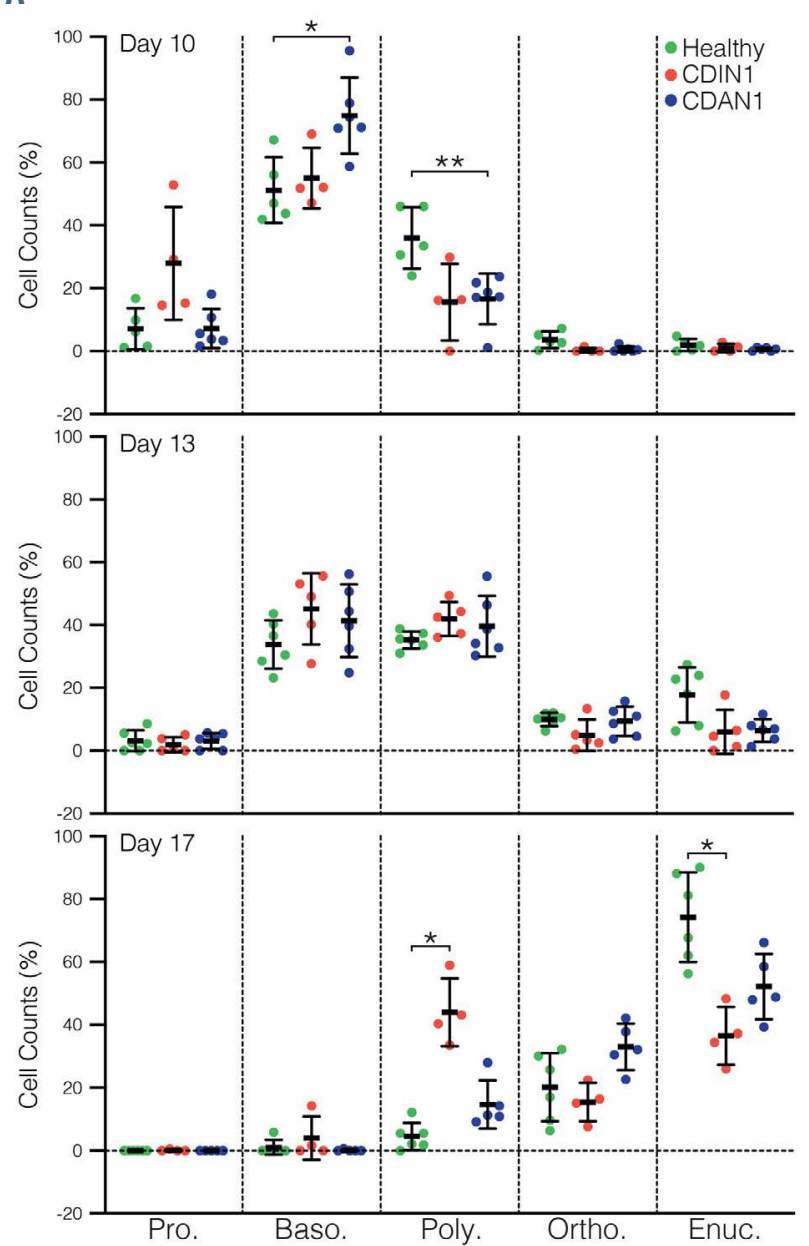

B

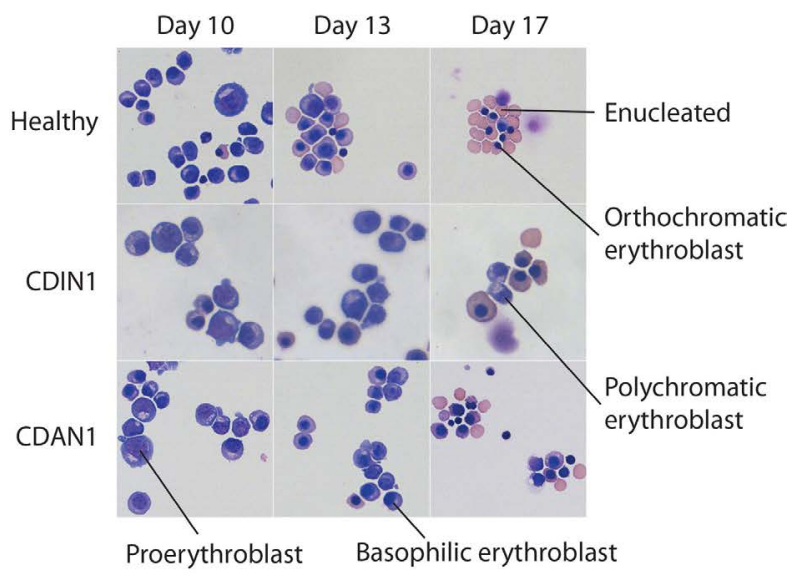

C

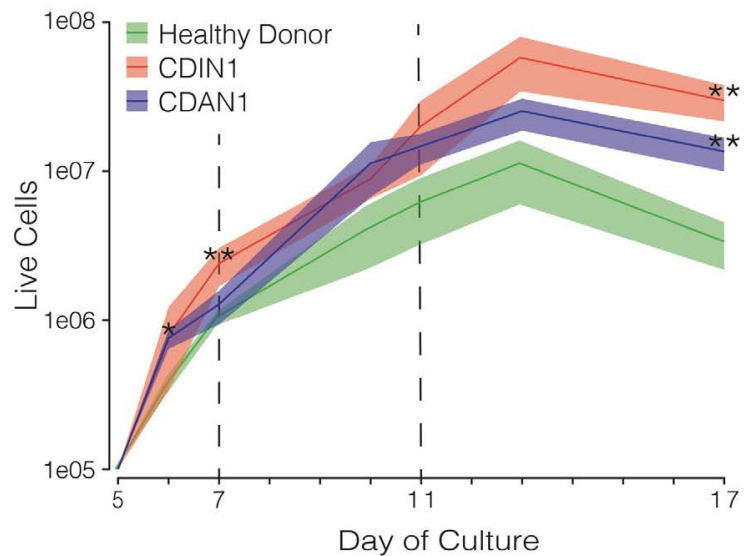

Figure 2. Erythroblasts from CDA-I patients display delayed differentiation and increased proliferation. (A) Cell morphology counts from cytospins on day 10 ( $n=101$ 348), day 13 ( $n=92-607)$ and day $17(n=63-329)$ of erythroblasts divided into proerythroblast (Pro), basophilic (Baso), polychromatic (Poly), orthochromatic (Ortho) and enucleated (Enuc). Data are shown as mean + standard deviation. Statistical significance was tested using Mann-Whitney with a Benjamani-Hochberg adjustment where $\mathrm{Q}=0.05$. CDIN1 patients and CDAN1 patients were tested separately against healthy donors for each timepoint. CDIN1 $* P=0.0095$ at day 17 for both polychromatic and enucleated erythroblasts. CDAN1 $* P=0.0087$ for day 10 basophilic erythroblasts and $* * P=0.0043$ for day 10 polychromatic erythroblasts. (B) Representative cytospin images stained with modified Wright's stain (magnification 40x) for healthy donors, CDIN1 and CDAN1 patients at days 10,13 and 17, with marked examples of cell types scored for (A). (C) Proliferation of cultured erythroblasts from healthy donors $(n=6)$, patients with mutations in $C D I N 1$ ( $n=3$ ) and patients with mutations in CDAN1 $(n=5)$ showing increased proliferation in both patient cohorts. All scores are for viable cells only (see Online Supplementary Figure S10), normalised to 100,000 cells at day 5. Data are shown as mean \pm standard error of the mean. Dashed vertical lines denote the three culture phases. Statistical significance was tested using Mann-Whitney with a Benjamani-Hochberg adjustment where $\mathrm{Q}=0.05$. CDIN1 patients and CDAN1 patients were tested separately against healthy donors for each timepoint. CDIN1 **P=0.0043 at day 7 and at day 17. CDAN1 $* P=0.0087$ at day 6 and $* * P=0.0043$ at day 17 . Cell counts from two patients were not scored in a comparable manner for this analysis.

gressed, cells visibly hemoglobinized by day 10 (Online Supplementary Figure S2D) and this coincided with increasing expression of the adult globins (Online Supplementary Figure $S 4 A$ ), with the $\alpha$ - to $\beta$-globin ratios remaining around one throughout differentiation (Online Supplementary Figure S4B). Iso-electric focusing (IEF) confirmed that predominantly adult globin was produced (Online Supplementary Figure S4C), and ATAC-seq showed open chromatin at the $H B A 1 / 2$, and $H B B$ genes and their associated locus control regions, again indicative of definitive erythropoiesis (Online Supplementary Figure $S 4 D$ to $E)$. During the third phase of culture, erythroblasts underwent the normal final stages of differentiation, with increased levels of Band 3 (CD233) and loss of the adhesion protein $\alpha-4$ integrin (CD49d) (Online Supplementary Figure S2C). Enucleated cells were visible on cytospins at this stage (Online Supplementary Figure S2B).

\section{Differentiating erythroblasts from healthy donors and CDA-I patients are broadly equivalent by immunophenotyping}

We studied the differentiation of erythroblasts derived from CD $34^{+}$HSPC from CDA-I patients with a variety of mutations in CDIN1 and CDAN1 (Online Supplementary Figure S1A). Flow cytometry bulk population analysis showed that differentiation of CDA-I patient HSPC appeared to be equivalent to that of the healthy donors with loss of $\mathrm{CD}_{3} 4^{+}$and gain of erythroid markers CD71 and CD235 from day 7, and expected changes in CD36, CD49d and Band 3 occurring from day 10. (Figure 1A, and for gating strategy see Online Supplementary Figure S3).

We also analysed the erythroblast immunophenotype by CyTOF, a next-generation flow cytometry platform that allows functional and phenotypic characterisation of cell populations. ${ }^{35}$ We examined the levels of 25 erythroid 
A

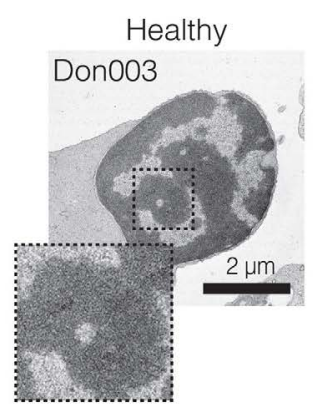

C

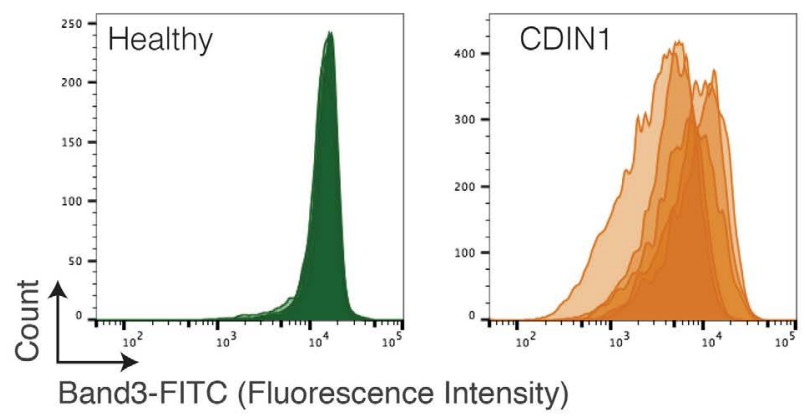

CDIN1
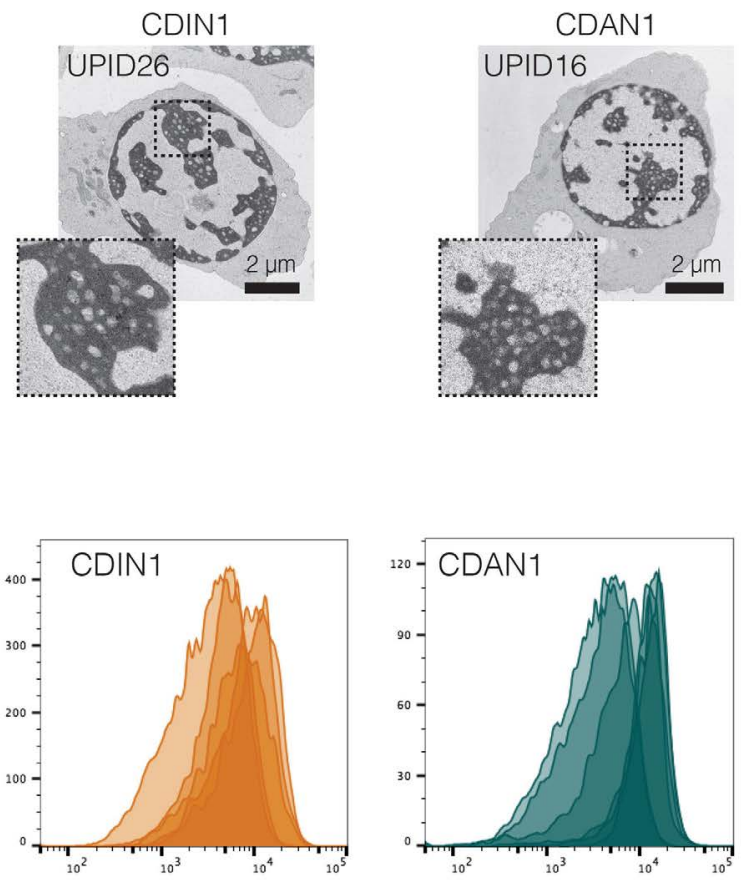

B

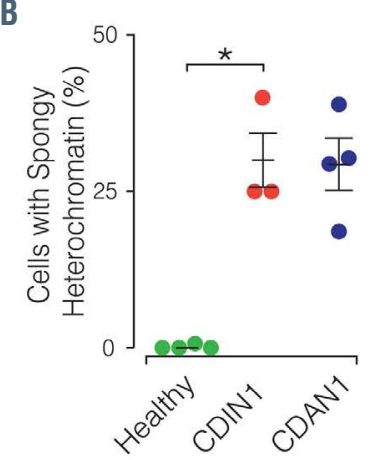

D

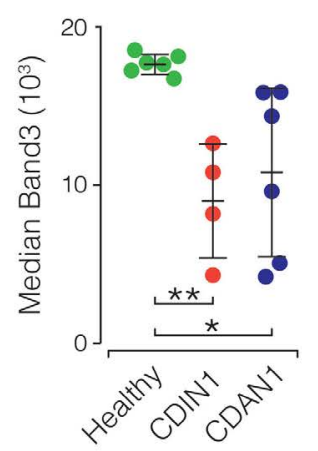

Figure 3. Evidence of defective differentiation in patient erythroblasts, most severe for CDIN1 mutations. (A) Transmission electron microscopy at day 11 of healthy and CDA-I cultured erythroblasts showing the diagnostic phenotype of abnormal chromatin in patients. Inset shows enlarged area to illustrate the pattern of euchromatin and heterochromatin and how this is disrupted in CDA-I patients. (B) Percentage of nuclei \pm standard deviation with spongy heterochromatin at day 11 , determined from large field images. Numbers of nuclei scored were $96-436$ per individual. ${ }^{*} P=0.0189$ with Kruskal-Wallis test. (C) Fluorescence activated cell sorting

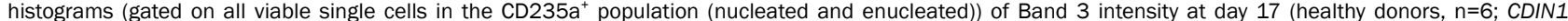
patients, $n=3$; and CDAN1 patients, $n=4$ ). (D) Median fluorescence intensity (MFI) \pm standard deviation of Band $3-F I T C$ at day 17 ( $* * P=0.0088$ and $* P=0.0127$ with Kruskal-Wallis test).

transcription factors and cell surface markers (Online Supplementary Table S6) at day 11 in healthy donor and CDIN1 patient-derived erythroblasts. Visualization by UMAP, widely used to identify distinct cell populations in cytometry data, ${ }^{28}$ revealed that erythroblasts from both groups follow a continuous trajectory during differentiation with a clear separation of cells expressing, for example, low levels of CD235 (denoted in blue) from those in the tail which have high expression of CD235 (Figure 1B). Cells from healthy donors and CDIN1 patients appear similarly distributed along the trajectory of differentiation at day 11 for all 25 markers analysed by CyTOF (Figure 1B; Online Supplementary Figure S5) and no significant differences in the clustering patterns were identified by Kmeans clustering analysis. ${ }^{36}$ Together with the FACS data (Figure 1A), this indicates that the patient and healthy donor samples cannot be distinguished by day $10 / 11$ on the basis of immunophenotype.

\section{Erythroblasts from CDA-I patients display delayed differentiation with increased proliferation}

Despite bulk population immunophenotyping indicating that patient and healthy donor cells are grossly stage matched in expansion and into differentiation, analysis of the proportions of morphologically-classed erythroid cells identified differences in the progression through differentiation, detectable from day 10 (Figure $2 \mathrm{~A}$ and $\mathrm{B}$ ). At this time point, significantly more erythroblasts from healthy donors than CDAN1 patients had reached the polychromatic stage. By day 17, there were more enucleated cells from healthy donors than CDIN1 patients, whose erythroblasts were still delayed at the polychromatic stage, with CDAN1 erythroblasts having progressed a little further. Therefore CDA-I patients exhibit clear delay in their erythroid differentiation. In order to firmly establish whether this represents a delay or a block, cultures would need to be continued beyond day 17 to look for persistence of precursor forms.

We also observed greater expansion in patient erythroblast numbers compared with healthy donors (Figure 2C) and this was significant even at the end of the expansion phase by day 6/7 (CDAN1, $P=0.0087$; CDIN1, $P=0.0043$ ). The increase in viable cell counts for patient erythroblasts became especially marked in the later phase of culture, reaching significance at day 17 of $P=0.0043$ for both patient groups.

\section{Evidence of defective differentiation in patient erythroblasts, most severe for CDIN1 mutations}

TEM revealed that the pattern of chromatin abnormalities characteristic of CDA-I was present in patient erythroblasts by day 11 of culture. This feature was observed in all our patient samples, averaging $29 \%$ ( \pm 7.7 standard deviation) of nuclei affected (Figures $3 \mathrm{~A}$ and $\mathrm{B}$; Online Supplementary Figure S7A). Furthermore, elevated expression of growth differentiation factor 15 (GDF15), a marker of ineffective erythropoiesis known to be increased in CDA-I patients, ${ }^{37}$ was detected at day 10 by immunofluorescence (IF) (Online Supplementary Figure S7B). Therefore, ex vivo differentiation of CDA-I erythroblasts successfully 
A

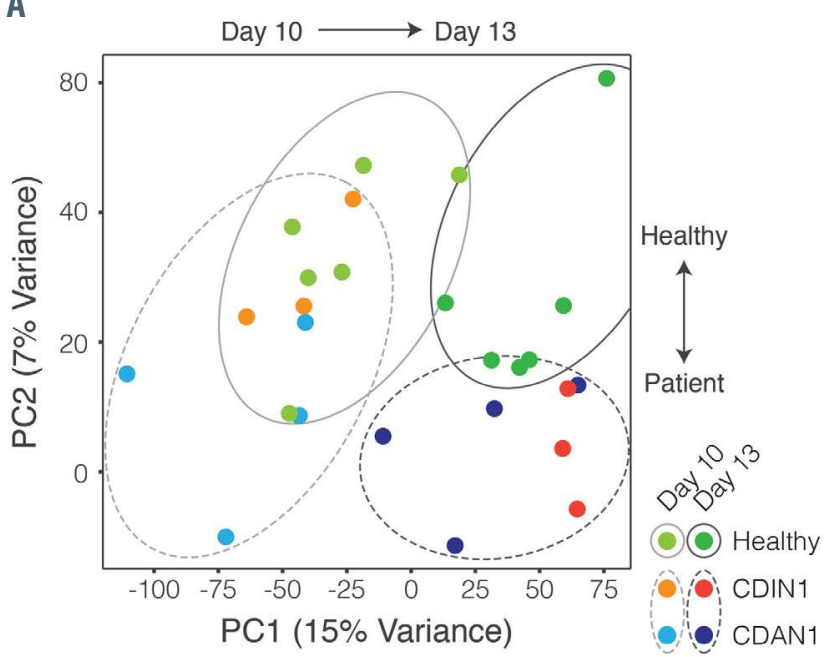

C
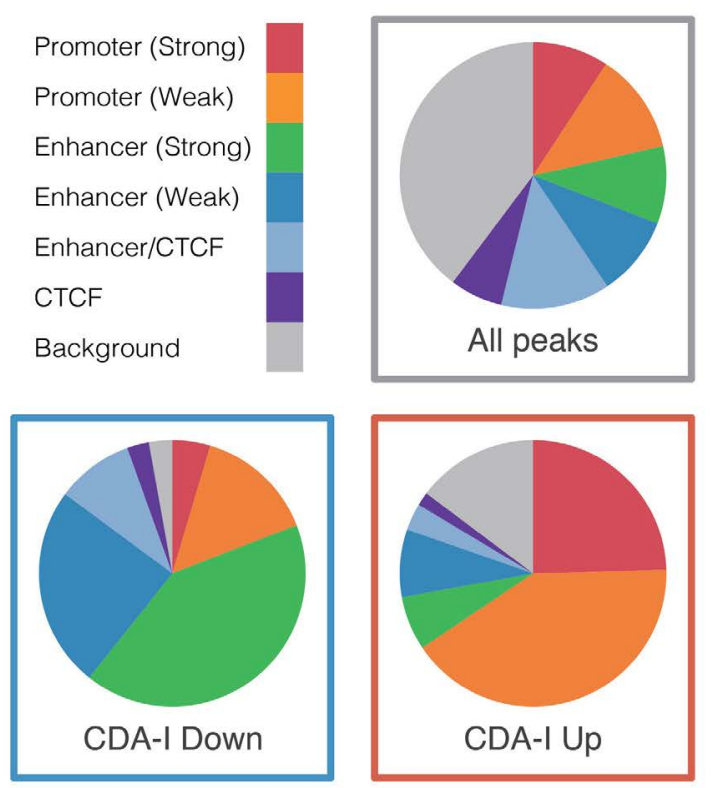

B

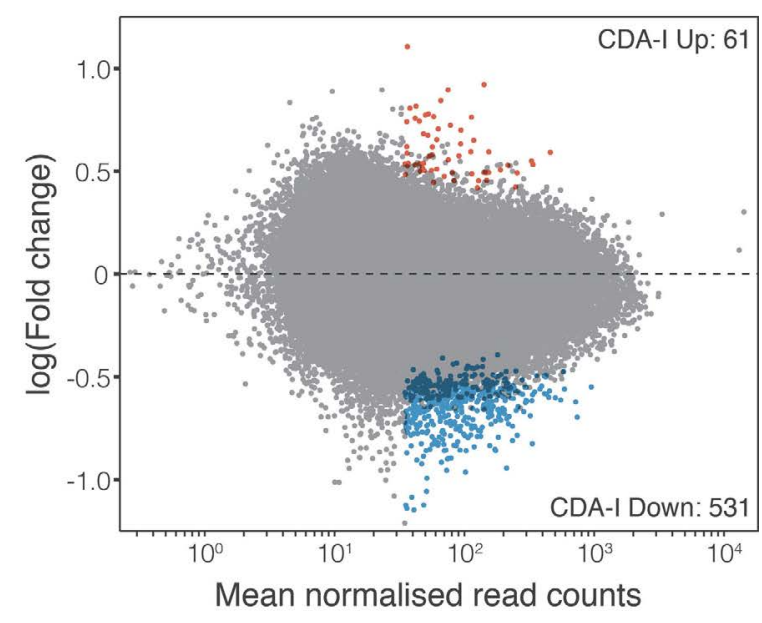

D
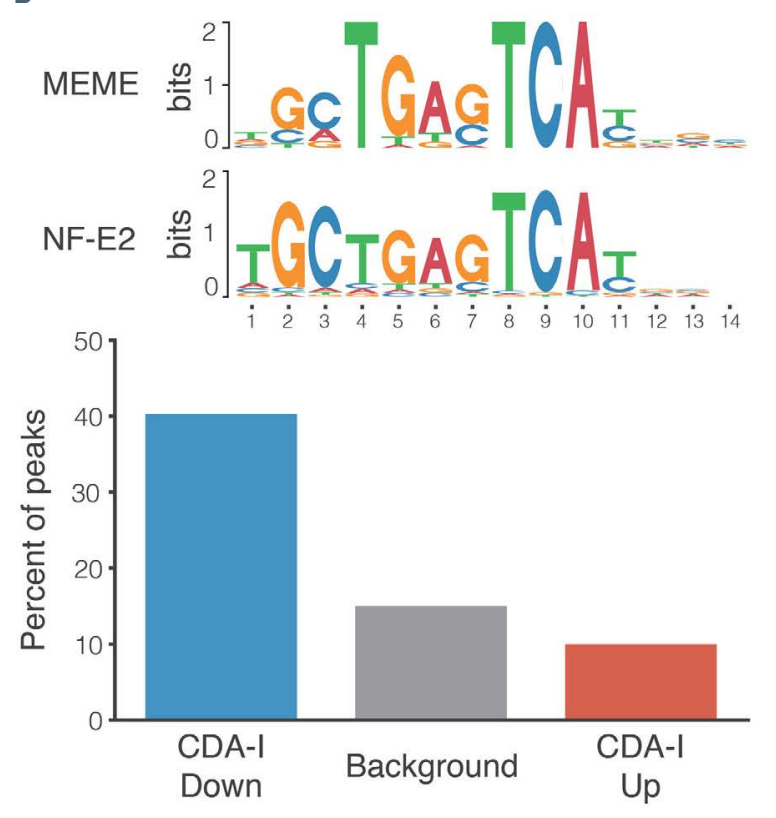

Figure 4. ATAC-seq analysis reveals the emergence of an altered regulatory landscape in patient-derived erythroblasts. (A) Principal component analysis (PCA) of assay for transposase-accessible chromatin using sequencing (ATAC-seq) from healthy donors $(n=6) C D I N 1$ patients $(n=3)$ and CDAN1 patients ( $n=4)$. The distribution of cells along PC1 follows differentiation stage and PC2 distinguishes patients and healthy donors. (B) MA plot for differential expression analysis (DESeq2) comparison of ATAC-seq from healthy donors and CDA-I patients at day 10 and 13 of ex vivo differentiation with significantly different peaks ( $q<0.01)$ highlighted as either more accessible in patients (red - up) or less accessible in patients (blue - down). (C) Comparison of chromatin state annotations ${ }^{26}$ for differentially accessible peaks shows enrichment for enhancers in less accessible peaks. Strong and weak refers to the level of H3K27ac signal. (D) MEME motif discovery identified a motif matching that of NF-E2 ${ }^{48,60}$ as being significantly enriched (E-value $<10^{-50}$ ) in ATAC-seq peaks that were less accessible in patients.

recapitulates abnormal cellular phenotypes observed in bone marrow derived erythroblasts and these features are already apparent midway through terminal differentiation.

We next assessed the effects of patient mutations on the enucleation stage of differentiation. Firstly, analysis of cellular morphology indicated a persistence of erythroid precursors in CDA-I cultures, particularly in those from patients with CDIN1 mutations, together with a significant reduction in the percentage of enucleated cells (Figure 2A). Secondly, immunophenotyping of cultured erythroblasts in the enucleation phase revealed changes in Band 3 expression in CDA-I patients. In normal erythropoiesis Band 3 shows a marked increase from the pro-erythrob- lasts to late erythroblasts, ${ }^{16}$ and while CDA-I patient erythroblasts did progressively gain Band 3, the level of protein was significantly less at day 17 than in healthy donors (Figure $3 \mathrm{C}$ and D). This supports other indications of a delay in the progression of differentiation in patientderived erythroblasts. Notably the Band 3 reduction was more severe in patients with CDIN1 mutations ( $\mathrm{n}=3$, $P=0.0088)$ than in the CDAN1 mutant cells $(\mathrm{n}=4$, $P=0.0127$ ) (Figure 3D).

\section{Differentiating erythroblasts from CDA-I patients display an altered regulatory landscape}

Analysis of open chromatin regions can be used to distinguish cell types and deconvolve mixed cell popula- 
A

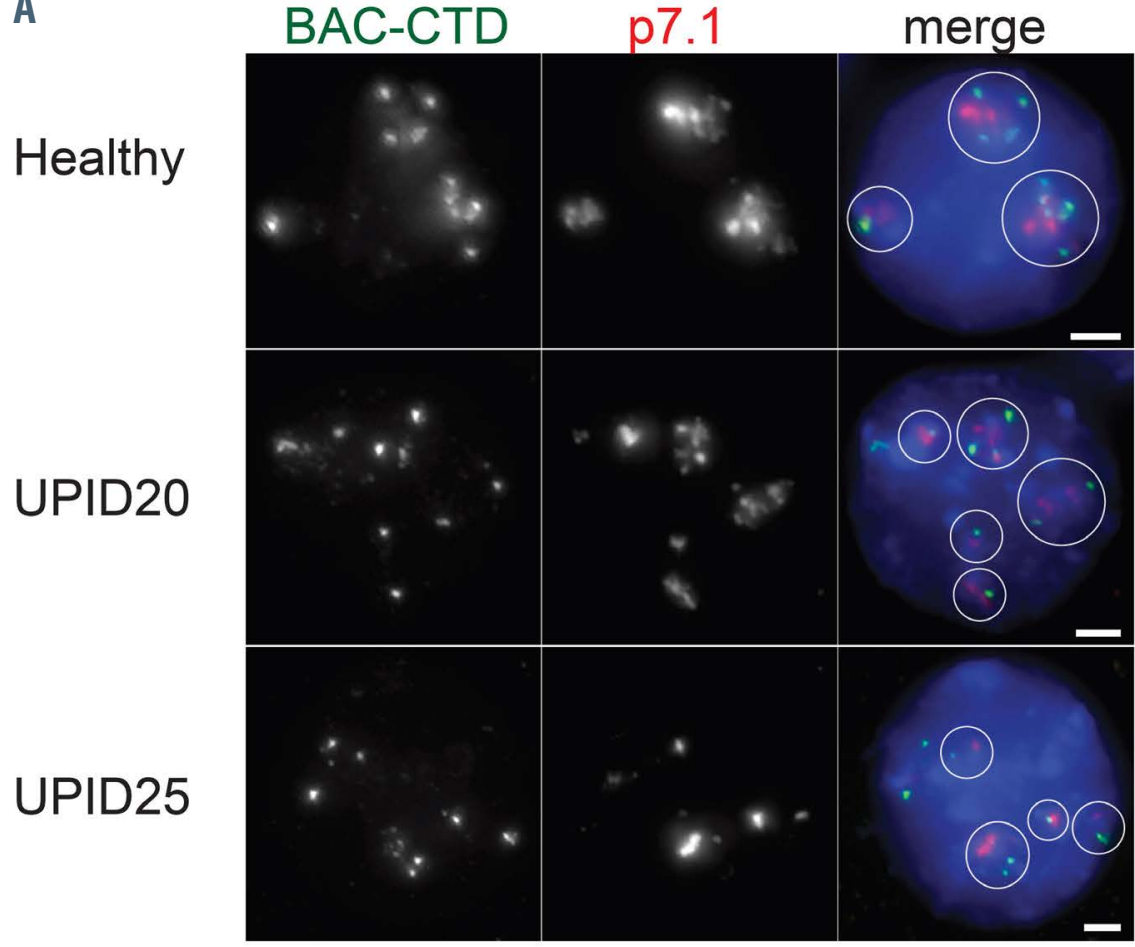

B

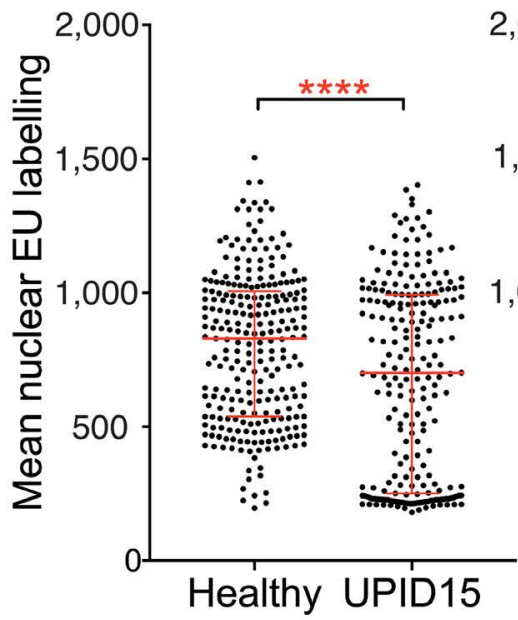

Figure 5. Nucleolar structure is disrupted in CDA-I patients. (A) Nucleoli detected with probe BAC-CTD (green) surrounding rDNA probe $\mathrm{p} 7.1$ (red) in healthy nuclei. In example patients UPID20 (CDAN1 mutation) and UPID25 (CDIN1 mutation) this order is disrupted. (B) Nucleolar output, judged by 5 ethynyl uridine (EU) labeling, is significantly reduced in two CDA-I patients with CDAN1 mutations, each compared with a healthy donor in the same experiment. (C) Despite the disrupted nucleolar structure, CDIN1 and Codanin-1 proteins (green) continue to associate with nucleolar proteins UBF and Fibrillarin (red) respectively, in two example CDA-I patients with CDAN1 mutations. All analyses are on day 10/11 cultured erythroblasts, using DAPI counterstain (blue).

C

\section{Healthy UPID22 UPID20}

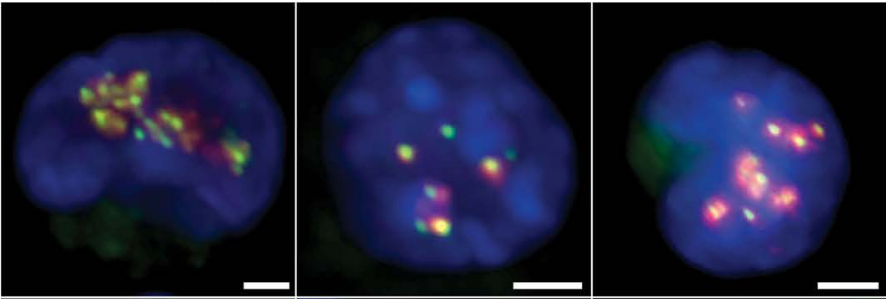

Codanin-1

Fibrillarin

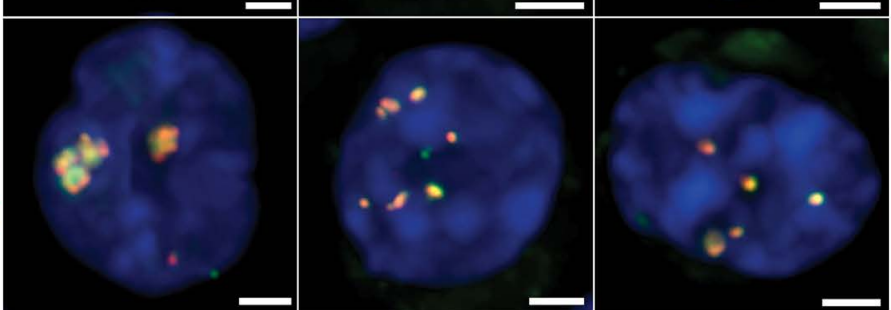


tions; ${ }^{18,38}$ therefore to further compare our differentiating healthy and patient cells we assayed chromatin accessibility at day 10 and day 13 using ATAC-seq. ${ }^{24}$ We mapped healthy and patient cell populations against a differentiation trajectory of sorted erythropoietic cell populations and 136,698 nucleosome-depleted regions, ${ }^{18,38}$ using principal component analysis (PCA) of all open chromatin regions (Online Supplementary Figure S6). This mapping could not distinguish patient material from healthy donors on the differentiation trajectory.

Next we undertook PCA of our cultured erythroblast data alone (without plotting against other erythroid populations), where a distinction in the accessibility profile between healthy donor and patient samples became apparent (Figure 4A), and this was more marked by day 13. We looked at pooled day 10 and day 13 ATAC-seq data by differential expression analysis (DESeq2) for differences in DNA accessibility peaks not attributable to differentiation status (Figure 4B). There were 61 peaks displaying increased accessibility in patients and 531 less accessible sites. The latter showed a marked enrichment for enhancers (strong and weak) (65\%) (Figure 4C) when assessed for chromatin state annotations. ${ }^{26}$ Furthermore, $40 \%$ of the 531 less accessible sites in CDA-I patients have a binding motif for the NF-E2 family of transcription factors (Figure 4D).

Consistent with motif distribution, the mean level of NF-E2 binding in ChIP-seq from healthy donor erythroblasts and K562 erythroleukemic cells was significantly higher than background at the peaks with patient-specific decreased accessibility (CDA-I down) (Online Supplementary Figure S8A), indicating that NF-E2 normally binds these sites. Any difference in NF-E2 binding could not be attributed to altered protein abundance as similar levels of NF-E2 were detected in healthy donors and CDIN1 patients by CyTOF mass cytometry (Online Supplementary Figure S8B). Similarly, no differences were seen in abundance for either MAFG, which dimerizes with NF-E2, ${ }^{39}$ or BACH1 which competitively binds with the NF-E2 motif. ${ }^{40,41}$

Of note, the Band 3 encoding gene Slc 4 a1 has erythroidspecific enhancer elements (Online Supplementary Figure S8C). The 5' enhancer has a binding motif for NF-E2 and is bound by NF-E2 in ChIP analyses of three different erythroblast cultures so that reduced accessibility at this site could account for reduced levels of Band 3 observed in CDA-I patients. The decrease in accessibility at this specific site did not quite reach significance in patient erythroblasts however the ATAC was performed at day 10 and day 13 which is possibly too early to observe an effect for this gene.

The structure of nucleoli is disrupted in CDA-I patients

With multiple strands of evidence for an altered pattern of differentiation in CDA-I patients, we looked for abnormal features that might be linked to the affected proteins. We have previously shown both Codanin-1 and CDIN1 endogenous proteins to be enriched in erythroblast nucleoli. ${ }^{10}$ Both proteins also show nucleolar enrichment in HEK293T (human embryonic kidney cell line), G-292 (human osteocarcinoma line), mES E14 (mouse embryonic stem cells) and B16F10 (mouse melanoma cell line) (data not shown), indicating this is a common feature across a range of cell types. We therefore examined nucleolar structure in day 10/11 erythroblasts by FISH using probes detecting the heterochromatic region adjacent to rDNA arrays (BAC-CTD), and the rDNA arrays themselves (p7.1) ${ }^{30}$ where the heterochromatin normally surrounds the rDNA signal within nucleoli. In all four patients examined (UPID10, 20, 22 and 25), nucleoli in a proportion of nuclei appeared more numerous, less ordered and less regular in shape, and the rDNA arrays appeared less open (Figure 5A). This was observed at a timepoint when $19-29 \%$ of nuclei in these patients exhibited abnormal chromatin distribution by TEM. Such disrupted organization might be expected to impact on the synthesis of ribosomal RNA, which accounts for a major portion of RNA synthesis in the cell. ${ }^{41,42}$ We therefore assessed RNA synthesis in two patients by measuring incorporation of the uridine analogue 5 ethynyl uridine (EU) into newly synthesised $\mathrm{RNA}^{42-44}$ at day 10/11 of differentiation. In both cases there is a significant reduction in nuclear EU labeling (Figure 5B) despite only a percentage of nuclei apparently affected and, particularly in UPID15, there is a distinct cell population with low EU signal. We have previously shown for four patients that the two mutated proteins are not destabilized by missense and in-frame mutations and remain detectable. ${ }^{10}$ Therefore, we investigated whether the normal enrichment of Codanin-1 and CDIN1 in nucleoli is also disrupted, in patients with predicted non-destabilizing mutations. Using IF with day 10 erythroblasts derived from CDAN1 patients UPID 6, 16, 20 and 22, in combination with nucleolar proteins UBF and Fibrillarin, we observed that the disrupted appearance of nucleoli recapitulated that observed by FISH (Figure 5C). Further, we were able to detect that both CDIN1 and the mutant Codanin-1 remained associated with nucleolar proteins in patient erythroblasts (Figure 5C).

\section{Using the culture system to validate novel variants in CDA-I patients}

The pathogenicity of novel CDIN1 or CDAN1 variants identified by sequencing requires further evidence, such as chromatin abnormalities in bone marrow biopsies by TEM. TEM is not only relatively inaccessible but often requires a second bone marrow biopsy. ${ }^{5}$ By contrast, peripheral blood is usually accessible and was used here in the ex vivo culture system to confirm the diagnosis of CDA-I in an infant with two novel mutations in CDAN1 (UPID33) (Online Supplementary Figure S1). ${ }^{10}$ Genetic analysis was conducted on the patient, who presented with unexplained anemia, using the Oxford Red Cell Panel (ORCP)45 (Online Supplementary Figure S9). Following identification of two novel variants, CD34 ${ }^{+}$ HSPC from UPID33 were extracted from peripheral blood and after 11 days in ex vivo culture, TEM on the resulting intermediate erythroblasts revealed $39 \%$ of erythroblasts with abnormal chromatin morphology, thus confirming diagnosis of CDA-I.

\section{Discussion}

Although understanding the cellular and molecular basis of CDA-I has the potential to elucidate new insights into the process of erythropoiesis, research is constrained by the limited studies that can be conducted using primary erythroid progenitors and precursors derived from patients with this condition. Here, using a modified exvivo culture system, we demonstrate that healthy control 
erythroblasts pass through the expected stages of differentiation with appropriate expression of erythroid cell surface markers ${ }^{16}$ and are able to enucleate. Further, we recapitulate the cardinal hematological features of CDA-I and show by TEM that up to $40 \%$ of patient-derived erythroblasts have spongy heterochromatin, indicating that ex vivo culture can be used to elucidate mechanisms underlying this disease.

We staged the cultures using an array of methods including FACS and CyTOF, which rely on immunophenotyping cell populations. While such methods showed healthy and diseased erythroblasts were immunophenotypically similar during the expansion phase and then into differentiation (day 10-13), aspects of disordered erythropoiesis were already evident at this mid-differentiation timepoint. In particular at this stage we noted a delay in progression through differentiation together with an increased proliferation of CDA-I erythroid precursors, producing increased amounts of GDF15, characteristic of dyserythropoiesis. ${ }^{37,46}$ Many of these cells exhibited the abnormal chromatin structure associated with CDA-I. These findings show that the effects of the mutant proteins start to operate early in terminal differentiation, indeed the viable cell counts would suggest that there may already be effects by the end of the expansion phase at day $6 / 7$.

Chromatin accessibility has become a superior approach for cell type classification, including hematopoietic lineages. ${ }^{38}$ The accessibility of transcription factor motifs within chromatin changes as subsets of regulatory elements are systematically activated and repressed during commitment to different lineages. ${ }^{47}$ Therefore, we used ATAC-seq as a genome-wide method to stage cell populations. When healthy donor and patient material from day 10 and day 13 cultures were aligned with a defined ATAC-seq erythroid trajectory, ${ }^{38}$ both map as expected with the intermediate and later stages of erythropoiesis. A more detailed PCA revealed a distinction between healthy donors and patients, more marked by day 13 , identified a reduced accessibility in patient erythroblasts at gene enhancers containing the erythroid-specific NF-E2 motif. NF-E2 (comprising NFE2-p45 and $\mathrm{MAFG}$ ) and $\mathrm{BACH} 1$ (which binds the same motif) are important transcription factors for erythropoiesis and the oxidative stress pathway respectively. ${ }^{99-41,48}$ CyTOF analysis indicates that levels of these three proteins appear to be normal in patient cells at day 11. NF-E2 motifs normally increase accessibility towards late erythropoiesis, ${ }^{18}$ in parallel with the level of the protein complex, 49,50 and we show that in normal erythroblasts, NF-E2 does bind those sites. Together these facts suggest that reduced accessibility of this motif at enhancers could affect the later stages of erythroid differentiation. It is possible that reduced accessibility of this motif reflects a generally altered regulatory landscape due to delayed differentiation, however motifs for other erythroid-specific transcription factors such as Gata1 did not reach significance in terms of altered accessibility.

The observation of disrupted nucleoli raises interesting ideas about the roles the two proteins may play in erythropoiesis and could explain the erythroid-specific nature of the disease. Mutations to the ribosomal proteins themselves can underlie tissue restricted disorders, including erythroid-specific disorders such as Diamond-Blackfan syndrome, Schwachmann-Diamond syndrome,
Dyskeratosis Congenita and MDS. ${ }^{43,51}$ Impaired function in the nucleolus could affect the number of available ribosomes and have similar effects to these other conditions in producing anemia. Beyond that, the nucleolus appears to have other, regulatory roles. ${ }^{43,52,53}$ Of interest, given the importance of cell cycle described above, is the proposed role for the nucleolus in cell cycle regulation. ${ }^{52}$ Another possibility is that CDIN1, with its sequence similarity to the Holliday junction resolvase family of proteins, could function in a repair pathway. The high transcription rate within nucleoli can lead to topological stress and double strand breaks ${ }^{53}$ whilst partial deletion of rDNA arrays has been shown to cause disordered nucleolar structure. ${ }^{54}$ Further work is required to test these possibilities.

The final stages of erythropoiesis involve nuclear condensation prior to expulsion of the pyknotic nuclei by enucleation ${ }^{55,56}$ and this process is highly organized ${ }^{50}$ with chromatin condensation playing an important role. ${ }^{57}$ The abnormal spongy heterochromatin observed in CDA-I could have a significant impact on the usual processes that precede enucleation, such as the selective loss of histones.$^{55}$ Remarkably, a substantial number of erythroblasts progress to enucleation without developing the catastrophic changes in chromatin compaction and organization apparent in spongy nuclei. This implies that the effects of the aberrant proteins must reach a threshold within individual cells to produce the pathognomonic phenotype and could be related to the balance between euchromatin and heterochromatin under nucleolar regulation. ${ }^{54,58}$

Two distinct types of CDA-I have been reported (CDAIa MIM 224120 and CDA-Ib MIM 615631) $)^{59}$ based on the levels of $\mathrm{Hb}$ and MCV, with the CDA-Ib patients (caused by CDIN1 mutations) thought to be more severely affected. In our patient cohort (excluding those regularly transfused or venesected), there is overlap between the blood indices irrespective of the mutation (Online Supplementary Figure S1). However, we observe a more pronounced delay in differentiation, increased proliferation and significantly reduced levels of Band 3 expression in erythroblasts cultured from CDIN1 patients, as compared to those with mutations in CDAN1. This implies that there may indeed be a distinction based on patient genotype where the CDA-I phenotype is more severe when arising from CDIN1 mutations.

In this study we provide a detailed characterization of CDA-I erythroblasts. We recapitulate aspects of the disease pathology seen in CDA-I, including high levels of cells with spongy heterochromatin and increased GDF15 expression. We report that CDA-I patient erythroblasts have elevated levels of proliferation, together with delay in the differentiation process and reduced levels of enucleation. There are difficulties in identifying and quantifying abnormalities in this disorder since only a proportion of erythroblasts exhibit defects whilst the majority differentiate and manage to function as red cells in many patients. Further, nurturing culture conditions may diminish the abnormal phenotypes observed. ${ }^{21}$ Nevertheless, ATACseq analysis provides clear evidence of an altered regulatory landscape during terminal differentiation. This, together with the observations of aberrant nucleolar structure and transcriptional output, gives insight into the underlying disease mechanism and highlights several new avenues for further investigation of the functional role of the two proteins in erythroid differentiation. 


\section{Disclosures}

No conflicts of interest to disclose.

\section{Contributions}

CS and DJD extracted CD $34^{+}$cells from CDA-I patient blood and normal donors respectively; $C S, D J D, M G$ performed the experiments with help from $A A O, R B, D J D$ and $R S$ undertook the ATAC analysis; DJPF and EJ performed the electron microscopy; JMB conducted the immunofluorescence, FISH and $E U$ labeling and analyzed the images; $C F$ and $A R$ conducted the $I E F ; M B$ conducted the CyTOF and the data was analyzed by $R B, Q A H, S O, R R, K R$ and $N R$ were the clinicians responsible for the care of the of the CDA-I patients; CS, DJD,JH,CB and $V J B$ conceived and designed experiments; DRH provided conceptual advice and clinical oversight; DJD and VJB created the figures; CS, DRH and VJB wrote the paper. All authors reviewed and critically edited the manuscript.

\section{Acknowledgments}

We thank the CDA-I patients for providing blood samples. We acknowledge the flow cytometry facility at the WIMM for providing cell analysis services and technical expertise, supported by the MRC HIU; MRC MHU (MC_UU_12009); NIHR Oxford BRC; Kay Kendall Leukemia Fund (KKL1057), John Fell Fund
(131/030 and 101/517), the EPA fund (CF182 and CF170) and by the WIMM Strategic Alliance awards G0902418 and MC_UU_12025. We also acknowledge the Electron Microscopy Facility at the Sir William Dunn School of Pathology for conducting the majority of the TEM and Raman Dhaliwal for help with sample preparation and imaging.

\section{Funding}

Further support came from grants to the Wolfson Imaging Center Oxford (Wolfson Foundation 18272, joint MRC/BBSRC/EPSRC MR/K015777X/1, Wellcome Trust Multi-User Equipment 104924/Z/14/Z). We would like to acknowledge Giorgio Napolitani and Michalina Mazurczyk for help in the mass cytometry facility at the WIMM, providing technical expertise and cell analysis services. The facility is supported by the MRC HIU core funded project MC_UU_00008 and the Oxford Single Cell Biology Consortium (OSCBC). This work was supported by the charity Congenital Anemia Network (CAN)(UK charity no. 1176864), Blood Buddies (UK charity no. 1108692), the Medical Research Council MC_UU_00016/1, a Wellcome Trust Strategic Award (106130/Z/14/Z) and the National Institute for Health research (NIHR) Oxford Biomedical Research Center Hematology Theme at Oxford University Hospitals NHS Trust and Oxford University.

\section{References}

1. Migliaccio AR, Varricchio L. Concise Review: advanced cell culture models for Diamond Blackfan anemia and other erythroid disorders. Stem Cells. 2018; 36(2):172-179.

2. Moniz H, Gastou M, Leblanc $\mathrm{T}$, et al. Primary hematopoietic cells from DBA patients with mutations in RPL11 and RPS19 genes exhibit distinct erythroid phenotype in vitro. Cell Death Dis. 2012 3(7):e356.

3. O'Brien KA, Farrar JE, Vlachos A, et al. Molecular convergence in ex vivo models of Diamond-Blackfan anemia. Blood. 2017; 129(23):3111-3120.

4. Renella R, Roberts NA, Brown JM, et al. Codanin-1 mutations in congenital dyserythropoietic anemia type 1 affect HP1alpha localization in erythroblasts. Blood. 2011; 117(25):6928-6938.

5. Roy NBA, Babbs C. The pathogenesis, diagnosis and management of congenital dyserythropoietic anaemia type I. $\mathrm{Br} \mathrm{J}$ Haematol. 2019;185(3):436-449.

6. Wickramasinghe SN. Congenital dyserythropoietic anaemias: clinical features, haematological morphology and new biochemical data. Blood Rev. 1998;12(3):178200.

7. Dgany O, Avidan N, Delaunay J, et al. Congenital dyserythropoietic anemia type I is caused by mutations in codanin-1. Am J Hum Genet. 2002;71(6):1467-1474.

8. Babbs C, Roberts NA, Sanchez-Pulido L, et al. Homozygous mutations in a predicted endonuclease are a novel cause of congenital dyserythropoietic anemia type I. Haematologica. 2013;98(9):1383-1387.

9. Swickley G, Bloch Y, Malka L, et al. Characterization of the interactions between Codanin-1 and C15Orf41, two proteins implicated in congenital dyserythropoietic anemia type I disease. BMC Mol Cell Biol. 2020;21(1):18.

10. Olijnik AA, Roy NBA, Scott C, et al.
Genetic and functional insights into CDA-I prevalence and pathogenesis. J Med Genet. 2021;58(3):185-195.

11. Shroff M, Knebel A, Toth R, Rouse J. A complex comprising C15ORF41 and Codanin-1- the products of two genes mutated in congenital dyserythropoietic anemia type I (CDA-I). Biochem J. 2020; 477(10):1893-1905

12. Noy-Lotan S, Dgany O, Lahmi R, et al. Codanin-1, the protein encoded by the gene mutated in congenital dyserythropoietic anemia type I (CDAN1), is cell cycleregulated. Haematologica. 2009;94(5):629637.

13. Ask K, Jasencakova Z, Menard P, Feng Y, Almouzni G, Groth A. Codanin-1, mutated in the anaemic disease CDAI, regulates Asf1 function in S-phase histone supply. EMBO J. 2012;31(8):2013-2023.

14. Ewing RM, Chu P, Elisma F, et al. Largescale mapping of human protein-protein interactions by mass spectrometry. Mol Syst Biol. 2007;3:89.

15. Griffiths RE, Kupzig S, Cogan N, et al. Maturing reticulocytes internalize plasma membrane in glycophorin A-containing vesicles that fuse with autophagosomes before exocytosis. Blood. 2012; 119(26):6296-6306.

16. Hu J, Liu J, Xue F, et al. Isolation and functional characterization of human erythroblasts at distinct stages: implications for understanding of normal and disordered erythropoiesis in vivo. Blood. 2013; 121(16):3246-3253

17. Mettananda S, Clark K, Fisher CA, SloaneStanley JA, Gibbons RJ, Higgs DR. Phenotypic and molecular characterization of a serum-free miniature erythroid differentiation system suitable for high-throughput screening and single-cell assays. Exp Hematol. 2018;60:10-20.

18. Ludwig LS, Lareau CA, Bao EL, et al. Transcriptional states and chromatin accessibility underlying human erythropoiesis. Cell Rep. 2019;27(11):3228-3240.
19. Satchwell TJ, Hawley BR, Bell AJ, Ribeiro ML, Toye AM. The cytoskeletal binding domain of band 3 is required for multiprotein complex formation and retention during erythropoiesis. Haematologica. 2015; 100(1):133-142

20. Satchwell TJ, Bell AJ, Hawley BR, et al. Severe Ankyrin-R deficiency results in impaired surface retention and lysosomal degradation of RhAG in human erythroblasts. Haematologica. 2016;101(9):10181027

21. Satchwell TJ, Pellegrin S, Bianchi P, et al. Characteristic phenotypes associated with congenital dyserythropoietic anemia (type II) manifest at different stages of erythropoiesis. Haematologica. 2013;98(11):17881796.

22. Ulirsch JC, Lareau C, Ludwig LS, Mohandas N, Nathan DG, Sankaran VG. Confounding in ex vivo models of Diamond-Blackfan anemia. Blood. 2017; 130(9):1165-1168.

23. Riffelmacher T, Clarke A, Richter FC, et al. Autophagy-dependent generation of free fatty acids is critical for normal neutrophil differentiation. Immunity. 2017;47(3):466480.

24. Buenrostro JD, $\mathrm{Wu}$ B, Litzenburger UM, et al. Single-cell chromatin accessibility reveals principles of regulatory variation. Nature. 2015;523(7561):486-490.

25. Hay D, Hughes JR, Babbs C, et al. Genetic dissection of the alpha-globin superenhancer in vivo. Nat Genet. 2016;48(8): 895-903.

26. Downes DJ, Schwessinger R, Hill SJ, et al. An integrated platform to systematically identify causal variants and genes for polygenic human traits. bioRxiv. 2020;813618.

27. Palii CG, Cheng Q, Gillespie MA, et al Single-Cell Proteomics reveal that quantitative changes in co-expressed lineage-specific transcription factors determine cell fate. Cell Stem Cell. 2019;24(5):812-820.

28. Becht E, McInnes L, Healy J, et al. Dimensionality reduction for visualizing 
single-cell data using UMAP. Nat Biotechnol. 2019;37:38-44.

29. Brown JM, Leach J, Reittie JE, et al. Coregulated human globin genes are frequently in spatial proximity when active. J Cell Biol. 2006;172(2):177-187.

30. Floutsakou I, Agrawal S, Nguyen TT, Seoighe C, Ganley AR, McStay B. The shared genomic architecture of human nucleolar organizer regions. Genome Res. 2013;23(12):2003-2012.

31. Brown J, Saracoglu K, Uhrig S, Speicher MR, Eils R, Kearney L. Subtelomeric chromosome rearrangements are detected using an innovative 12-color FISH assay (M-TEL). Nat Med. 2001;7(4):497-501.

32. Schindelin J, Arganda-Carreras I, Frise E, et al. Fiji: an open-source platform for biological-image analysis. Nat Methods. 2012; 9(7):676-682

33. Heimpel H, Schwarz K, Ebnother M, et al. Congenital dyserythropoietic anemia type I (CDA I): molecular genetics, clinical appearance, and prognosis based on longterm observation. Blood. 2006;107(1):334340.

34. Heimpel H, Kellermann K, Neuschwander N, Hogel J, Schwarz K. The morphological diagnosis of congenital dyserythropoietic anemia: results of a quantitative analysis of peripheral blood and bone marrow cells. Haematologica. 2010;95(6):1034-1036.

35. Kay AW, Strauss-Albee DM, Blish CA. Application of mass cytometry (CyTOF) for functional and phenotypic analysis of natural killer cells. Methods Mol Biol. 2016:1441:13-26.

36. MacQueen J. Some methods for classification and analysis of multivariate observations. Proc Fifth Berkley Sympon Math Statist and Prob. 1967;1:281-297.

37. Tamary H, Shalev H, Perez-Avraham G, et al. Elevated growth differentiation factor 15 expression in patients with congenital dyserythropoietic anemia type I. Blood. 2008;112(13):5241-5244.

38. Corces MR, Buenrostro JD, Wu B, et al. Lineage-specific and single-cell chromatin accessibility charts human hematopoiesis and leukemia evolution. Nat Genet. 2016;48(10):1193-1203

39. Blank V, Kim MJ, Andrews NC. Human MafG is a functional partner for p45 NF-E2 in activating globin gene expression. Blood. 1997;89(11):3925-3935.

40. Oyake T, Itoh K, Motohashi $\mathrm{H}$, et al. Bach proteins belong to a novel family of BTBbasic leucine zipper transcription factors that interact with MafK and regulate transcription through the NF-E2 site. Mol Cell Biol. 1996;16(11):6083-6095.

41. Sun J, Brand M, Zenke Y, Tashiro S, Groudine M, Igarashi K. Heme regulates the dynamic exchange of Bach1 and NF-E2related factors in the Maf transcription factor network. Proc Natl Acad Sci U S A. 2004;101(6):1461-1466.

42. Jao CY, Salic A. Exploring RNA transcription and turnover in vivo by using click chemistry. Proc Natl Acad Sci U S A. 2008; 105(41):15779-15784

43.Bohnsack KE, Bohnsack MT. Uncovering the assembly pathway of human ribosomes and its emerging links to disease. EMBO J. 2019;38(13):e100278.

44. Warner JR. The economics of ribosome biosynthesis in yeast. Trends Biochem Sci. 1999;24(11):437-440.

45. Roy NB, Wilson EA, Henderson S, et al. A novel 33-Gene targeted resequencing panel provides accurate, clinical-grade diagnosis and improves patient management for rare inherited anaemias. Br J Haematol. 2016; 175(2):318-330

46. Tanno T, Noel P, Miller JL. Growth differentiation factor 15 in erythroid health and disease. Curr Opin Hematol. 2010; 17(3):184-190.

47. Heuston EF, Keller CA, Lichtenberg J, et al. Establishment of regulatory elements during erythro-megakaryopoiesis identifies hematopoietic lineage-commitment points. Epigenetics Chromatin. 2018;11(1):22.

48. Andrews NC, Kotkow KJ, Ney PA, Erdjument-Bromage $\mathrm{H}$, Tempst P, Orkin $\mathrm{SH}$. The ubiquitous subunit of erythroid transcription factor NF-E2 is a small basicleucine zipper protein related to the v-maf oncogene. Proc Natl Acad Sci U S A. 1993;90(24):11488-11492

49. Gillespie MA, Palii CG, Sanchez-Taltavull $\mathrm{D}$, et al. Absolute quantification of transcription factors reveals principles of gene regulation in erythropoiesis. Mol Cell. 2020;78(5):960-974

50. Gautier EF, Ducamp S, Leduc M, et al Comprehensive proteomic analysis of human erythropoiesis. Cell Rep. 2016; 16(5):1470-1484.

51. Nakhoul H, Ke J, Zhou X, Liao W, Zeng SX, $\mathrm{Lu} \mathrm{H}$. Ribosomopathies: mechanisms of disease. Clin Med Insights Blood Disord. 2014;7:7-16.

52. Andersen JS, Lyon CE, Fox AH, et al Directed proteomic analysis of the human nucleolus. Curr Biol. 2002;12(1):1-11.

53. Tsekrekou M, Stratigi K, Chatzinikolaou G. The nucleolus: in genome maintenance and repair. Int J Mol Sci. 2017;18(7):1411.

54. Paredes S, Maggert KA. Ribosomal DNA contributes to global chromatin regulation. Proc Natl Acad Sci U S A. 2009; 106(42): 17829-17834.

55. Zhao B, Mei Y, Schipma MJ, et al. Nuclear condensation during mouse erythropoiesis requires Caspase-3-mediated nuclear opening. Dev Cell. 2016;36(5):498-510.

56. Baron MH, Barminko J. Chromatin condensation and enucleation in red blood cells: an open question. Dev Cell. 2016;36(5):481482.

57. Ney PA. Normal and disordered reticulocyte maturation. Curr Opin Hematol. 2011; 18(3):152-157.

58. Guetg C, Lienemann P, Sirri V, et al. The NoRC complex mediates the heterochromatin formation and stability of silent rRNA genes and centromeric repeats. EMBO J. 2010;29(13):2135-2146

59. Gambale A, Iolascon A, Andolfo I, Russo R. Diagnosis and management of congenital dyserythropoietic anemias. Expert Rev Hematol. 2016;9(3):283-296.

60. Daftari P, Gavva NR, Shen CK. Distinction between AP1 and NF-E2 factor-binding at specific chromatin regions in mammalian cells. Oncogene. 1999;18(39):5482-5486. 